\title{
Bead Capture on Magnetic Sensors in a Microfluidic System
}

Østerberg, Frederik Westergaard; Dalslet, Bjarke Thomas; Damsgaard, Christian Danvad; Freitas, S. C.; Freitas, P. P.; Hansen, Mikkel Fougt

Published in:

IEEE Sensors Journal

Link to article, DOI:

10.1109/JSEN.2009.2021122

Publication date:

2009

Document Version

Publisher's PDF, also known as Version of record

Link back to DTU Orbit

Citation (APA):

Østerberg, F. W., Dalslet, B. T., Damsgaard, C. D., Freitas, S. C., Freitas, P. P., \& Hansen, M. F. (2009). Bead Capture on Magnetic Sensors in a Microfluidic System. IEEE Sensors Journal, 9(5-6), 682-688.

https://doi.org/10.1109/JSEN.2009.2021122

\section{General rights}

Copyright and moral rights for the publications made accessible in the public portal are retained by the authors and/or other copyright owners and it is a condition of accessing publications that users recognise and abide by the legal requirements associated with these rights.

- Users may download and print one copy of any publication from the public portal for the purpose of private study or research.

- You may not further distribute the material or use it for any profit-making activity or commercial gain

- You may freely distribute the URL identifying the publication in the public portal 


\title{
Bead Capture on Magnetic Sensors in a Microfluidic System
}

\author{
Frederik W. Østerberg, Bjarke T. Dalslet, Christian D. Damsgaard, Susana C. Freitas, Paulo P. Freitas, and \\ Mikkel F. Hansen
}

\begin{abstract}
The accumulation of magnetic beads by gravitational sedimentation and magnetic capture on a planar Hall-effect sensor integrated in a microfluidic channel is studied systematically as a function of the bead concentration, the fluid flow rate, and the sensor bias current. It is demonstrated that the sedimentation flux is proportional to the bead concentration and has a power law relation to the fluid flow rate. The mechanisms for the bead accumulation are investigated and it is found that gravitational sedimentation dominates the bead accumulation, whereas the stability of the sedimented beads against the fluid flow is defined by the localized magnetic fields from the sensor.
\end{abstract}

Index Terms-AMR, bead, biosensor, lab-on-a-chip, microfluidic.

\section{INTRODUCTION}

$\mathbf{M}$ AGNETIC biosensors relying on magnetic detection of magnetic beads bound to the sensor surface by a bioassay show great potential for being compact biosensors with a direct electrical readout (for reviews, see [1]-[5]). Several magnetic sensor principles are being explored, e.g., giant magnetoresistance and spin valve sensors [3]-[7], magnetic tunnel junctions [3], [4], micro-Hall sensors [8], and planar Hall effect (PHE) sensors [9]-[11].

Common for all sensor principles is that the biological sample and the magnetic bead suspension is introduced to the sensor followed by washing steps. To achieve reproducible conditions under these various incubation and washing steps, it is desirable to integrate the sensor in a fluidic system. To design this system, it is important to know how the experimental conditions influence the results. Previously, the sedimentation of beads from a stagnant bead suspension on a Wheatstone configuration of spin-valve sensors in a trench has been studied by Thilwind et al. [7].

Manuscript received February 20, 2009; revised March 23, 2009; accepted March 27, 2009. Current version published May 01, 2009. This work was supported in part by the EU Sixth Framework Program BIODIAGNOSTICS Project under Contract 017002 and in part by The Danish Research Council for Technology and Production Sciences under Grant 274-06-0454. This is an expanded paper from the Sensors 2008 Conference. The associate editor coordinating the review of this paper and approving it for publication was Dr. Patrick Ruther.

F. W. Østerberg, B. T. Dalslet, C. D. Damsgaard, and M. F. Hansen are with the Department of Micro and Nanotechnology, Technical University of Denmark (DTU Nanotech), DK-2800 Kongens, Lyngby, Denmark (e-mail: S042421@student.dtu.dk; bjarke.dalslet@nanotech.dtu.dk; christian.damsgaard@nanotech.dtu.dk; mikkel.hansen@nanotech.dtu.dk).

S. C. Freitas and P. P. Freitas are with the INESC-microsystems and nanotechnologies, Rue Alves Redol, 9-1, 1000-029 and Instituto Superior Tecnico, Physics Department, Avenida Rovisco Pais, 1000 Lisboa, Portugal (e-mail: sfreitas@inesc-mn.pt; pfreitas@inesc-mn.pt).

Color versions of one or more of the figures in this paper are available online at http://ieeexplore.ieee.org.

Digital Object Identifier 10.1109/JSEN.2009.2021122
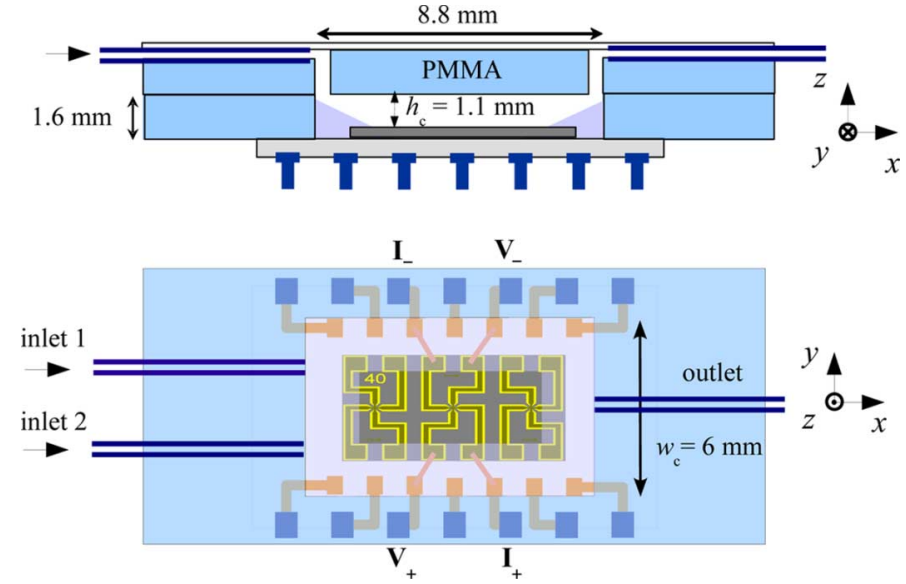

Fig. 1. Schematic cross-sectional and top views of the fluid system defined in PMMA and attached to the ceramic chip carrier. The connections for the bias current and the measured voltage are indicated by $I_{-}, I_{+}$and $V_{-}, V_{+}$, respectively, for the middle sensor.

In this study, we focus on the interaction between magnetic beads in a flowing suspension and the sensor surface of PHE sensors. Using the electromagnetic field from the bias current passed through the sensor, we systematically study the real-time response of PHE sensors integrated in a microfluidic channel exposed to a bead suspension as function of the time $t$, the bead concentration $c$, the fluid flow rate $Q$ and the sensor bias current $I_{x}$. The experimental studies are supplemented by theoretical calculations and a discussion of the bead accumulation mechanisms.

\section{EXPERIMENTAL}

\section{A. System Design and Fabrication}

The exchange-biased PHE sensors with the structure $\mathrm{Si} / \mathrm{SiO}_{2}(800 \mathrm{~nm}) / \mathrm{Ta}(5 \mathrm{~nm}) / \mathrm{Ni}_{81} \mathrm{Fe}_{19}(30 \mathrm{~nm}) / \mathrm{Mn}_{76} \mathrm{Ir}_{24}(20 \mathrm{~nm}) /$ $\mathrm{Ta}(5 \mathrm{~nm})$ were deposited by ion beam deposition as described in [12] and subsequently defined by ion milling. During deposition, a magnetic flux density of $4 \mathrm{mT}$ was applied to define an easy magnetization direction of the permalloy layer in the positive $x$-direction (see Fig. 1). Gold contacts were deposited by e-beam evaporation and defined by liftoff. Subsequently, a $470 \mathrm{~nm}$ protective layer of $\mathrm{SiO}_{2}$ was $\mathrm{RF}$ sputter deposited through a shadow mask. A sensor chip had three sensor crosses that each had an active area of $w \times w=40 \mu \mathrm{m} \times 40 \mu \mathrm{m}$. The chip was mounted on and wire bonded to a ceramic chip carrier. During experiments, a current $I_{x}$ was applied through the sensors in the $x$-direction and the voltage drop $V_{y}$ along the $y$-direction was measured as indicated for the middle sensor 


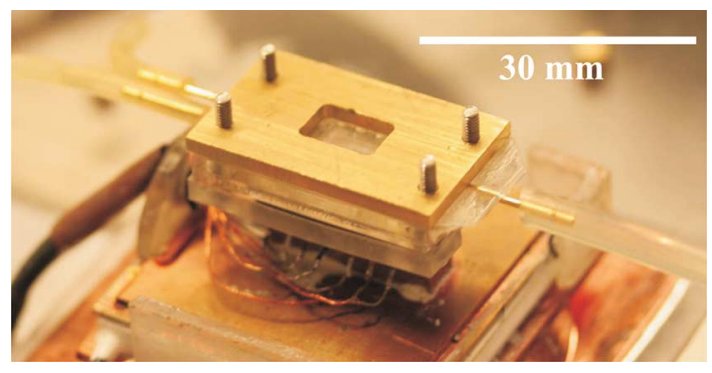

Fig. 2. The assembled fluid system on the Peltier element-based temperature control system.

in Fig. 1. For low fields $H_{y}$ along the $y$-direction, the sensor output is

$$
V_{y}=I_{x} S_{0} H_{y}
$$

where $S_{0}$ is the sensor sensitivity [10]. The sensitivity for the sensors used in the experiments was found to $6.2 \times 10^{-5} \mathrm{~V} \mathrm{~m} / \mathrm{A}^{2}$.

A fluid system was assembled around the sensor and substrate as sketched in Fig. 1. The fluid system was defined by laser cutting in PMMA and consisted of a 2-mm-thick fluid plate defining the fluid chamber glued to the ceramic chip carrier and a top with fluid inlets and outlet. The channels on the top part were sealed by lamination foil. The wire bonds were protected with silicone glue (Elastosil E41, Wacker Chemie, München, Germany). This glue also sealed the interface between the ceramic chip carrier and the fluid chamber plate. The two-part fluid system was sealed by an O-ring. The channel cross section at the chip was $w_{\mathrm{c}} \times h_{\mathrm{c}}=6 \mathrm{~mm} \times 1.1 \mathrm{~mm}$. The system had two inlets to allow for an easy switching between bead suspension and water.

To ensure a constant temperature during experiments, the system was mounted on a $\mathrm{Cu}$ block with integrated thermometer and temperature control by use of a Peltier element. Fig. 2 shows the system mounted in the experimental setup with temperature control.

\section{B. Experimental Procedure}

The two fluid inlets were connected to syringes with MyOne bead suspension and deionized (DI) water, respectively. The fluid flow rates were controlled by Harvard Apparatus PHD 2000 syringe pumps. An ac sensor bias current with a frequency of $2200 \mathrm{~Hz}$ was supplied by a Keithley 6221 current source. After preamplification in a Stanford Research Systems (SRS) SR552 bipolar preamplifier, the signal was extracted by use of a SRS SR830 lock-in amplifier. During all experiments, the sample holder temperature was kept constant at $28^{\circ} \mathrm{C}$. All measurements were carried out in zero applied magnetic field and without any magnetic shielding.

All experiments and calculations were carried out with MyOne beads, which have a diameter of $D=1.05 \mu \mathrm{m}$, a density of $\rho_{\mathrm{b}}=1700 \mathrm{~kg} / \mathrm{m}^{3}$ and a measured magnetic susceptibility of $\chi=1.377$ [13].

Prior to experiments, the fluid system was flushed with DI water to remove air bubbles. Then, a syringe with freshly mixed bead suspension of mass-to-volume concentration $c$ was mounted in the syringe pump and the bead suspension was injected at a flow rate $Q$. The responses of all three sensors were monitored versus the time $t$ after the bead suspension entered the fluid system. Subsequently, the fluid system was cleaned by letting an air bubble into the channel followed by rinse with DI water. It was verified that the sensor signals returned to their baseline level after washing. A comparison of the signals from the three sensors as well as repeated experiments verified that the experimental observations were reproducible.

\section{THEORY}

\section{A. Sensor Response to Self-Field}

We consider the sensor response for an ac applied current

$$
I_{x}(t)=I_{x, 0} \sin (\omega t)
$$

in zero external magnetic field. In the absence of magnetic beads, the sensor is still affected by an average field in the $y$-direction because part of the applied current is shunted in the antiferromagnetic $\mathrm{Mn}_{76} \mathrm{Ir}_{24}$ layer. We write this field as $\gamma_{0} I_{x}$, where $\gamma_{0}$ is a constant related to the sensor stack and geometry. When the antiferromagnet is deposited on top of the permalloy layer, the field from a current passing through the antiferromagnetic layer in the positive $x$-direction gives rise to a field acting on the ferromagnetic layer in the positive $y$-direction and hence $\gamma_{0}$ is positive. When beads are present on the sensor, they will also give rise to a positive field in the $y$-direction, which we write as $\gamma_{1} I_{x}$ [14]. Hence, the sensor signal due to its self-magnetization and beads can be written as

$$
V_{y}=I_{x, 0}^{2} \sin ^{2}(\omega t) S_{0}\left(\gamma_{0}+\gamma_{1}\right) .
$$

The signal is detected by a lock-in amplifier. The lock-in amplifier multiplies $V$ with a reference signal $V_{\text {ref }}(t)=$ $\sqrt{2} \sin (n \omega t+\phi)$, where $n$ is the harmonic chosen for the detection and $\phi$ is the phase of the reference signal $(\phi=0$ for detection of the in-phase response and $\phi=\pi / 2$ for detection of the out-of-phase response). Subsequently, the signal is passed through a low-pass filter. The measurements in the present study are all carried out using the second harmonic out-of-phase response ( $n=2, \phi=\pi / 2$ ), which is calculated to

$$
V_{y, 2}^{\prime \prime}=-\frac{1}{2 \sqrt{2}} S_{0} I_{x, 0}^{2}\left(\gamma_{0}+\gamma_{1}\right) \text {. }
$$

Thus, assuming that $\gamma_{1}$ is proportional to the bead coverage, we can use measurements of $V_{y, 2}^{\prime \prime}$ to monitor the amount of beads in the vicinity of the sensor.

\section{B. Bead Sedimentation}

We consider the motion of a bead in a fluid flow under the influence of buoyancy, the Stokes fluid drag force and magnetic forces. The equation of motion for a single bead with the coordinate system defined in Fig. 1 is

$$
\mathbf{F}_{\text {bead }}=\mathbf{F}_{\text {buoy }}-3 \pi \eta D\left(\mathbf{v}_{\mathrm{b}}-\mathbf{v}_{\mathrm{f}}\right)+\mathbf{F}_{\text {mag }}
$$

where $\mathbf{F}_{\text {buoy }} \equiv-\hat{\mathbf{z}} g \frac{\pi}{6} D^{3}\left(\rho_{\mathrm{b}}-\rho_{\mathrm{f}}\right), \hat{\mathbf{z}}$ is the unit vector in the $z$-direction, $g=9.82 \mathrm{~m} / \mathrm{s}^{2}$ is the magnitude of the gravitational 


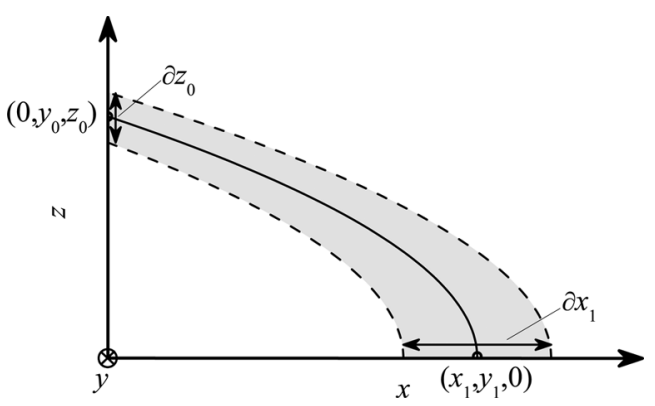

Fig. 3. Cross section of the fluidic channel. The figure illustrates a bead trajectory, as well as the infinitesimal volumes around the trajectory.

acceleration, $\rho_{\mathrm{f}}=1.0 \times 10^{3} \mathrm{~kg} / \mathrm{m}^{3}$ is the density of water, $\eta=8.2 \times 10^{-4}$ Pas is the viscosity of water and $\mathbf{v}_{\mathrm{b}}$ and $\mathbf{v}_{\mathrm{f}}$ are the bead and fluid velocities, respectively. In this equation, we have neglected bead-bead interactions and interactions between the bead and the channel wall. Hence, it is assumed that the beads are ideal tracer particles for the flow.

First, we analytically calculate the sedimentation in the absence of magnetic forces. Second, we consider the magnetic force arising from the bias current. We will base all numbers on a bead mass-volume concentration of $c=0.1 \mathrm{~kg} / \mathrm{m}^{3}$ corresponding to a bead number concentration of $c_{\mathrm{n}}=9.7 \times 10^{13}$ beads $/ \mathrm{m}^{3}$.

1) Bead Sedimentation Flux in the Absence of Magnetic Forces: By assumption, the only forces in the $z$-direction are the drag and buoyancy forces. It is found from (5) that

$$
v_{z}=\frac{D^{2}\left(\rho_{\mathrm{f}}-\rho_{\mathrm{b}}\right) g}{18 \eta} .
$$

The bead velocity $v_{x}$ along the channel is equal to the fluid flow velocity. We assume that the fluid velocity is only nonzero in the $x$-direction and only depends on $y$ and $z$, i.e., $v_{x}=v_{x}(y, z)$. As no forces act in the $y$-direction, $v_{y}=0$. Fig. 3 shows a bead trajectory and the definition of coordinates used in the calculations.

The number of beads entering the channel per time through an infinitesimal area $\partial y_{0} \partial z_{0}$ in the $y z$-plane at $\left(0, y_{0}, z_{0}\right)$ is

$$
\frac{\partial N}{\partial t}=v_{x}\left(y_{0}, z_{0}\right) c_{\mathrm{n}} \partial y_{0} \partial z_{0}
$$

These beads land in the $x y$-plane on the infinitesimal area $\partial x_{1} \partial y_{1}$ around $\left(x_{1}, y_{1}, 0\right)$. Hence, the number of beads landing per area per time (the bead sedimentation flux) is

$$
\frac{\partial N}{\partial t \partial x_{1} \partial y_{1}}=v_{x}\left(y_{0}, z_{0}\right) c_{\mathrm{n}} \frac{\partial y_{0}}{\partial y_{1}} \frac{\partial z_{0}}{\partial x_{1}} \text {. }
$$

Since the velocity in the $y$-direction is zero, $y_{0}=y_{1}$ and $\left(\partial y_{0} / \partial y_{1}\right)=1$. To find $\partial z_{0} / \partial x_{1}$, we need the relation between $\partial z_{0}$ at the starting coordinates $\left(0, y_{0}, z_{0}\right)$ and $\partial x_{1}$ at the landing coordinates $\left(x_{1}, y_{0}, 0\right)$. The time $t_{0}$ it takes for a bead to sediment from an initial height $z_{0}$ over the channel bottom is $t_{0}=-z_{0} / v_{z}$. The distance traveled by the bead in the $x$-direction is

$$
\begin{aligned}
x_{1} & =\int_{0}^{t_{0}} v_{x}\left(y_{0}, z(t)\right) d t \\
& =-v_{z}^{-1} \int_{0}^{z_{0}} v_{x}\left(y_{0}, z\right) d z .
\end{aligned}
$$

Hence

$$
\begin{aligned}
\frac{\partial z_{0}}{\partial x_{1}} & =\left(\frac{\partial x_{1}}{\partial z_{0}}\right)^{-1} \\
& =\frac{-v_{z}}{v_{x}\left(y_{0}, z_{0}\right)}
\end{aligned}
$$

which inserted in (8) results in the bead sedimentation flux

$$
\frac{\partial N}{\partial t \partial x_{1} \partial y_{1}}=-v_{z} \cdot c_{\mathrm{n}} \text {. }
$$

Thus, we have shown that the bead sedimentation flux is uniform and independent of the fluid flow velocity profile. Inserting the numbers for our MyOne bead suspension yields a bead sedimentation flux of $4.98 \times 10^{7} \mathrm{~s}^{-1} \mathrm{~m}^{-2}$. For our sensor, which has an active area of $40 \times 40 \mu \mathrm{m}^{2}$, this corresponds to one bead landing on the sensor every $12.6 \mathrm{~s}$.

To get a feel for the bead coverage, it is also convenient to express the bead sedimentation flux in terms of the number $N_{\mathrm{ML}}$ of monolayers of close-packed beads on the surface. Using that each bead takes up the area $(\sqrt{3} / 2) D^{2}$ and (11) and (6), we find

$$
N_{\mathrm{ML}}(t)=\frac{\sqrt{3}}{6 \pi} \frac{\rho_{\mathrm{b}}-\rho_{\mathrm{w}}}{\rho_{\mathrm{b}}} \frac{g D}{\eta} \cdot t c .
$$

Inserting the values for MyOne beads we find $v_{z}=-0.51 \mu \mathrm{m} / \mathrm{s}$ and that $N_{\mathrm{ML}}=4.76 \times 10^{-4} \mathrm{~m}^{3} \mathrm{~kg}^{-1} \mathrm{~s}^{-1} \cdot t c$. For a bead concentration of $c=0.1 \mathrm{~kg} / \mathrm{m}^{3}$ this means that a monolayer of beads should sediment in about $21.0 \mathrm{ks}$.

2) Magnetic Forces: In this section we consider the magnetic force on a bead arising from the bias current. The magnetic force is given by [15]

$$
\mathbf{F}_{\text {mag }} \simeq \frac{1}{12} \pi D^{3} \mu_{0} \chi \nabla\left(\mathbf{H}^{2}\right)
$$

where $\mu_{0}$ is the permeability of free space and $\mathbf{H}$ is the magnetic field intensity in the absence of the magnetic bead. This field has contributions due to the bias current passed through the sensor and the magnetostatic field from the ferromagnetic $\mathrm{Ni}_{81} \mathrm{Fe}_{19}$ layer. In our study, we will restrict ourselves to study the former contribution. For an infinitely long surface conductor of width $w=40 \mu \mathrm{m}$ placed along the $x$ axis centered at $(x, 0,0)$, the field at a point $(x, y, z)$ is found from Biot and Savart's law as

$$
\begin{aligned}
\mathbf{H}=\frac{I_{x}}{2 \pi w}(\arctan ( & \left.\frac{-4 w z}{4 z^{2}+4 y^{2}-w^{2}}\right) \hat{\mathbf{y}} \\
& \left.+\frac{1}{2} \ln \left(\frac{(y+w / 2)^{2}+z^{2}}{(y-w / 2)^{2}+z^{2}}\right) \hat{\mathbf{z}}\right) .
\end{aligned}
$$

When inserting this into (13), an analytical expression for the magnetic force is obtained. This expression is then evaluated in the space around the conductor slap. A contour plot of the $z$-component of the magnetic force divided by the buoyancy force is shown in Fig. 4 for the typical ac current amplitude of $I_{x, 0}=7.1 \mathrm{~mA}$ corresponding to a root mean square (rms) current of $5 \mathrm{~mA}$. The magnetic force is found to be smaller than the buoyancy for all bead positions and is thus expected not to be important for the bead capture. As $F_{\text {mag, }}$ is proportional to $I_{x, 0}^{2}$, contours for other currents can easily be found by adding $2 \log _{10}\left(I_{x, 0} / 1 \mathrm{~mA}\right)$ to the contour values in Fig. 4. Even for an eight times higher current amplitude of $I_{x, 0}=56.8 \mathrm{~mA}, F_{\text {mag,z }}$ 


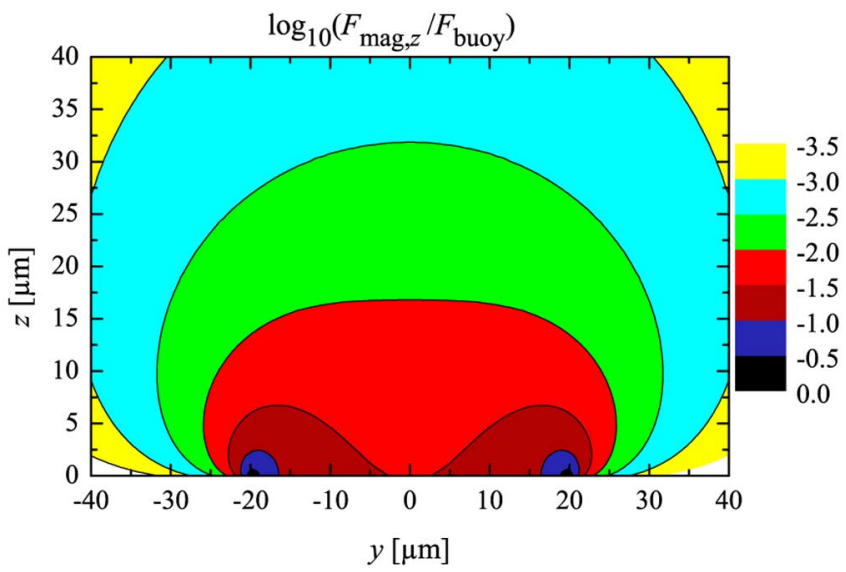

Fig. 4. Contour plot of the $z$-component of the average magnetic force divided by the buoyancy force. The magnetic force is calculated for an ac current with amplitude $I_{x, 0}=7.1 \mathrm{~mA}$.

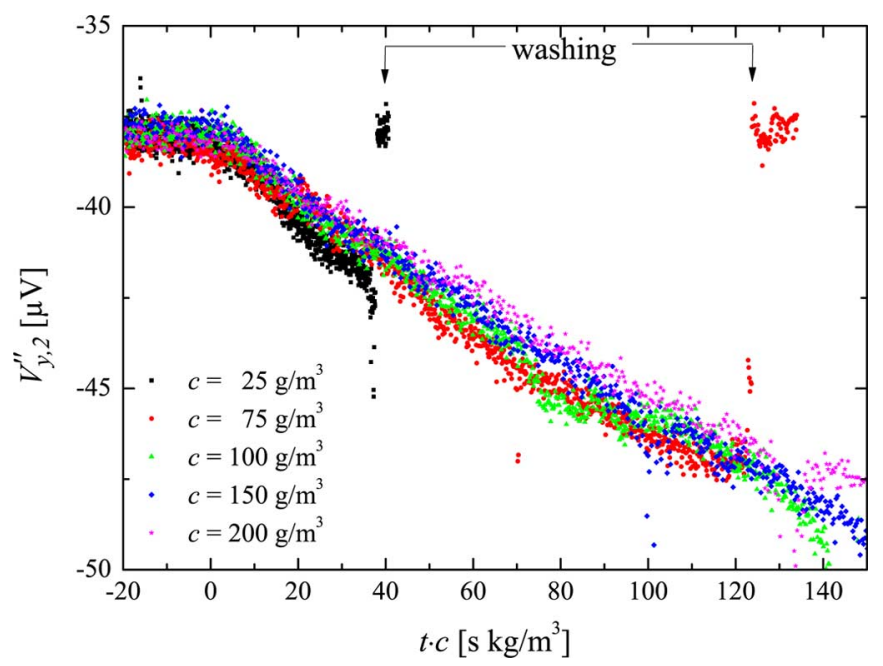

Fig. 5. Values of $V_{y, 2}^{\prime \prime}$ measured as function of $t \cdot c$ for the indicated bead concentrations $c$. Fixed values of $Q=50 \mu \mathrm{L} / \mathrm{min}$ and $I_{x, 0}=7.1 \mathrm{~mA}$ were used in the experiments.

will only be larger than $F_{\text {buoy }}$ up to $15 \mu \mathrm{m}$ in the $z$-direction over the sensor edges. This distance is small compared to the channel height suggesting that gravitational sedimentation will dominate the accumulation of beads.

\section{RESULTS}

Three measurement series were performed as a function of the bead concentration $c$, the fluid flow rate $Q$, and the bias current amplitude $I_{x, 0}$, where one parameter was varied, while the others were kept constant.

\section{A. Bead Concentration Dependence}

The bead concentration was varied between $c=0.025 \mathrm{~kg} / \mathrm{m}^{3}$ and $c=0.2 \mathrm{~kg} / \mathrm{m}^{3}$ for fixed $Q=50 \mu \mathrm{L} / \mathrm{min}$ and $I_{x, 0}=7.1 \mathrm{~mA}$. Fig. 5 shows measured values of the second harmonic out-ofphase response $V_{y, 2}^{\prime \prime}$ as a function of $t \cdot c$, where $t$ is the time after the bead suspension is injected into the channel. It is observed that the time traces of $V_{y, 2}^{\prime \prime}$ for different bead concentrations in

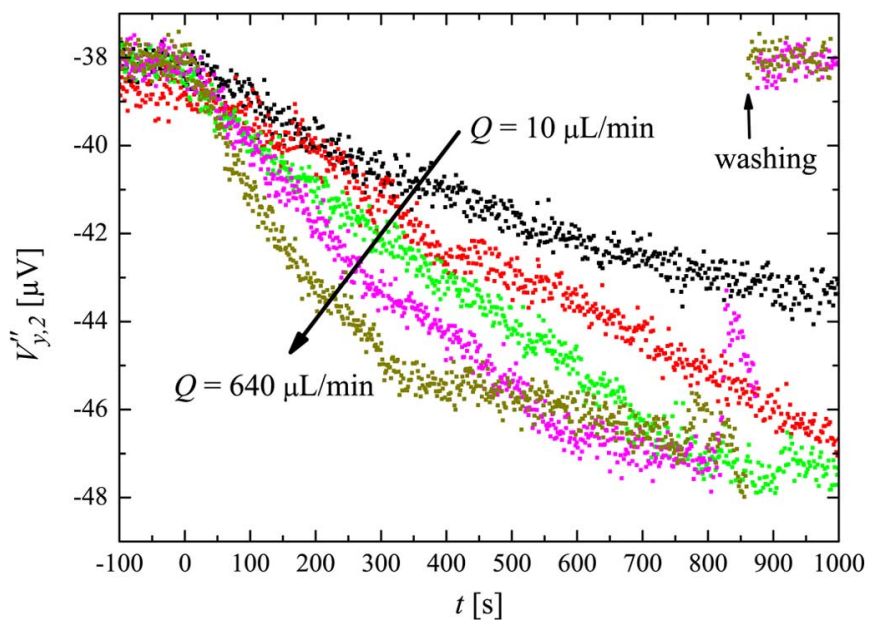

Fig. 6. Values of $V_{y, 2}^{\prime \prime}$ measured as function of time, for $Q=10,20,40,160$, and $640 \mu \mathrm{L} / \mathrm{min}$. Fixed values of $c=0.1 \mathrm{~kg} / \mathrm{m}^{3}$ and $I_{x, 0}=7.1 \mathrm{~mA}$ were used in the experiments.

Fig. 5 fall onto the same curve. Moreover, the time traces of $V_{y, 2}^{\prime \prime}$ are linear.

For $c=0.025 \mathrm{~kg} / \mathrm{m}^{3}$ and $c=0.075 \mathrm{~kg} / \mathrm{m}^{3}$, the bead suspension flow was switched off after some time and an air bubble was injected followed by DI water to wash the beads out of the fluid system. In both cases, the signal is observed to return to its baseline level.

\section{B. Flow Rate Dependence}

The flow rate was varied between $Q=10 \mu \mathrm{L} / \mathrm{min}$ and $Q=$ $640 \mu \mathrm{L} / \mathrm{min}$ for fixed $c=0.1 \mathrm{~kg} / \mathrm{m}^{3}$ and $I_{x, 0}=7.1 \mathrm{~mA}$. Fig. 6 shows measured values of the second harmonic out-of-phase response $V_{y, 2}^{\prime \prime}$ as a function of $t$.

For small $t>0$, the signal changes linearly with time as was also observed in Fig. 5. The initial slope, $-\partial V_{y, 2}^{\prime \prime} / \partial t$, varies systematically with $Q$ and increases for increasing $Q$. For longer times, the signals level off and approach their saturation values. The time at which this happens is shorter for high values of $Q$.

Fig. 7 shows the time derivatives $-\partial V_{y, 2}^{\prime \prime} / \partial t$ of the initial response in Fig. 6 as function of $Q$. The inset in Fig. 7 shows the same data on a $\log -\log$ scale. It is seen that the data points are well described by a power law in the investigated interval. A fit yields the expression

$$
-\frac{\partial V_{y, 2}^{\prime \prime}}{\partial t}=2.9 \frac{\mathrm{nV}}{\mathrm{s}} \cdot(Q[\mu \mathrm{L} / \mathrm{min}])^{0.36}
$$

which is shown as the lines in figure.

In a preliminary study in [16] using the same fluidic system and bead type with $c=0.2 \mathrm{~kg} / \mathrm{m}^{3}$ and a different sensor from the same wafer, the initial slope of the sensor response was found to have a linear dependence on $Q$ within the significant experimental uncertainty. In the present study, the fluid handling was improved resulting in more reproducible experimental results.

\section{Bias Current Dependence}

To further investigate the capture of magnetic beads on the sensor, we studied the dependence of the measured sensor re- 


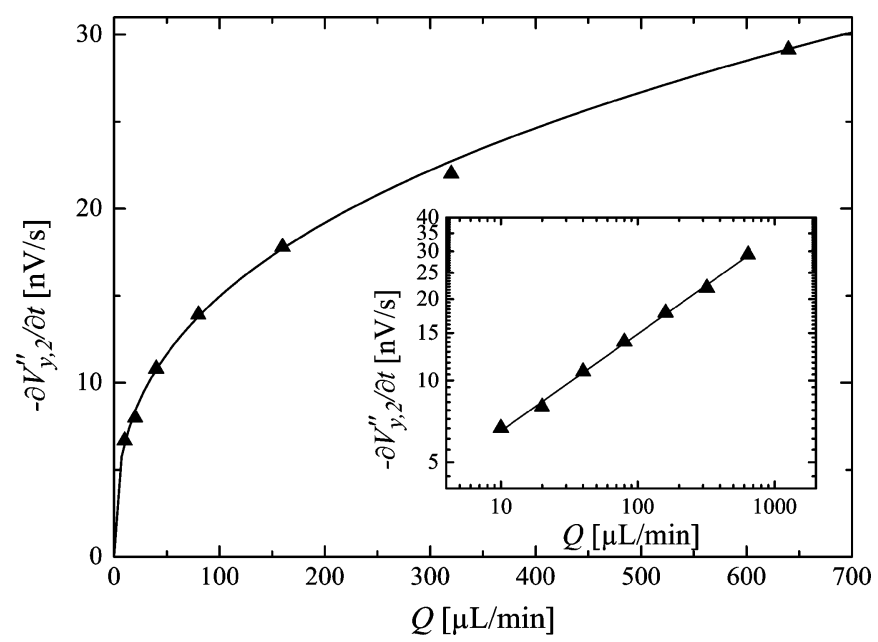

Fig. 7. Minus the initial slope of the plots in Fig. 6 plotted as a function of $Q$ on lin-lin scale and on log-log scale (inset). The line is a power law fit.

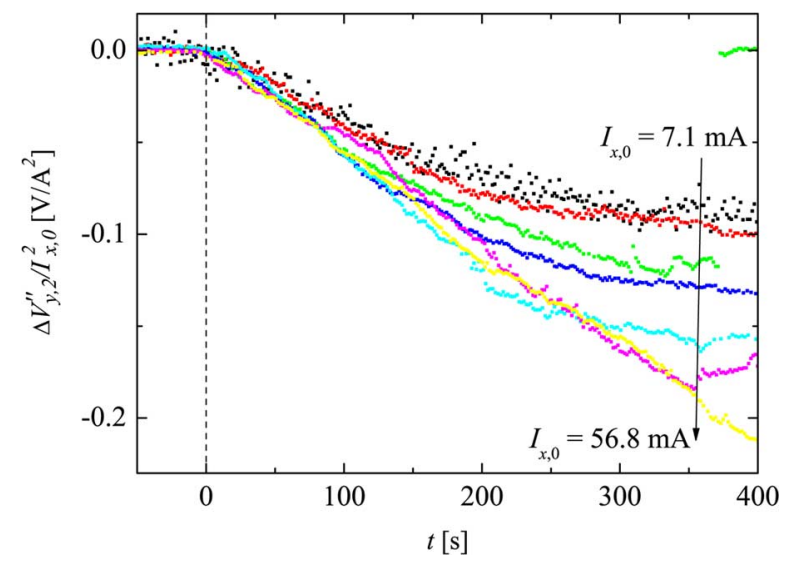

Fig. 8. Normalized change in sensor response $\Delta V_{y, 2}^{\prime \prime} / I_{x, 0}^{2}=\left(V_{y, 2}^{\prime \prime}(t>0)-\right.$ $\left.V_{y, 2}^{\prime \prime}(t<0)\right) / I_{x, 0}^{2}$ measured for $I_{x, 0}=7.1,14.2,21.3,28.4,42.6,49.7$, and $56.8 \mathrm{~mA}$. Fixed values of $c=0.1 \mathrm{~kg} / \mathrm{m}^{3}$ and $Q=320 \mu \mathrm{L} / \mathrm{min}$ were used in the experiments.

sponse on the bias current. The current amplitude was varied from $I_{x, 0}=7.1 \mathrm{~mA}$ to $I_{x, 0}=56.8 \mathrm{~mA}$ for constant $c=0.1$ $\mathrm{kg} / \mathrm{m}^{3}$ and $Q=320 \mu \mathrm{L} / \mathrm{min}$. The increasing current resulted in some self-heating of the sensor. To focus on the response due to the beads, we therefore plot $\Delta V_{y, 2}^{\prime \prime} / I_{x, 0}^{2} \equiv\left(V_{y, 2}^{\prime \prime}(t>\right.$ $\left.0)-V_{y, 2}^{\prime \prime}(t<0)\right) / I_{x, 0}^{2}$ in Fig. 8, which according to (4) is directly proportional to the bead signal.

It is seen that the slopes of the normalized signals approximately assume the same value for all values of $I_{x, 0}$. However, for each value of $I_{x, 0}$, the time, where the signal levels off and approaches a saturation value, increases with increasing value of $I_{x, 0}$. Moreover, the absolute saturation value increases with increasing $I_{x, 0}$.

\section{DISCUSSION}

In the experiments where the bead concentration was varied for fixed values of $Q$ and $I_{x, 0}$, the signal was found to be a function of $t \cdot c$ and increase linearly with $t$. Both of these results are in agreement with the predictions of the theoretical analysis of the gravitational bead sedimentation in (12). According

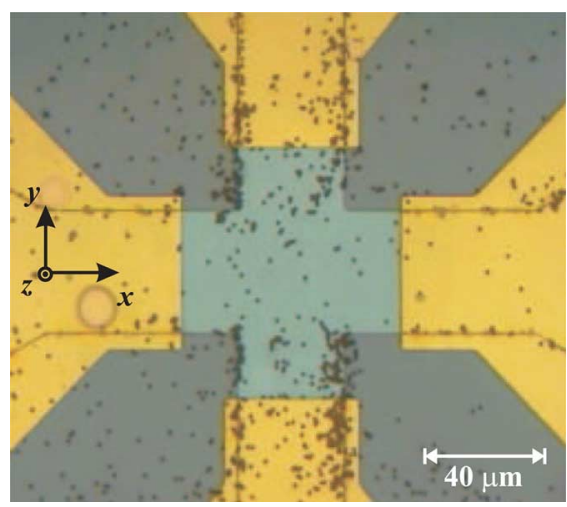

Fig. 9. Micrograph of sensor after exposure to beads and gentle washing. The permalloy layer is magnetized in the $x$-direction. Beads are clearly observed to accumulate near edges with nonzero normal magnetization values.

to this analysis less than a single monolayer of beads should be sedimented on average on the surface for all experimental conditions. Thus, the observed linear change in the signal is consistent with a linearly increasing partial coverage of the sensor assuming that the sensor response is proportional to the bead coverage.

To discuss the signal dependence on $Q$, we first consider the effect of varying $Q$ on the gravitational sedimentation of beads. According to the theoretical calculations in (12), the influx of magnetic beads on the surface is constant. When it is taken into account that the fluid flow can also remove beads from the surface and that the probability of a bead being removed increases with increasing $Q$, one would expect zero or a negative change of $-\partial V_{y, 2}^{\prime \prime} / \partial t$ with increasing $Q$. Hence, the observations in Fig. 7 cannot be explained in terms of just gravitational sedimentation of the beads.

The magnetic force on a bead due to the applied current is strongly localized near the edges of the conductor (cfr. Section III-B2). The magnetic forces will be able to capture and hold beads passing close to the conductor even when $Q$ is large enough to tear sedimented beads away from areas in the channel with no magnetic forces. The contour defining the bead capture zone, i.e., the region where the magnetic force is strong enough to prevent beads from being torn loose by the flow, depends on both $I_{x, 0}$ and $Q$.

The magnetostatic field from the $\mathrm{Ni}_{81} \mathrm{Fe}_{19}$ layer can also create capture zones as it gives rise to a force on the beads in the vicinity of the $\mathrm{Ni}_{81} \mathrm{Fe}_{19}$ layer edges with a nonzero normal magnetization component. That this effect plays a role can be observed in Fig. 9, which shows a micrograph of a dry sensor after exposure to the magnetic bead suspension and gentle washing. The beads are clearly observed to accumulate near the edges of the $\mathrm{Ni}_{81} \mathrm{Fe}_{19}$ layer parallel with the $y$ axis where there is a significant magnetostatic field due to the sensor magnetization in the $x$-direction.

The sensor signal from accumulating beads will initially be determined by the amount of beads entering capture zones on and near the sensor. Beads can either be captured from the flowing suspension by the short range magnetic forces or while rolling along the surface. Hence, $-\partial V_{y, 2}^{\prime \prime} / \partial t$ will grow with increasing $Q$, as observed in Fig. 6. 
When sufficiently many layers of beads have accumulated in the capture zones, beads will begin to tear loose as the magnetic forces in the top layers are too weak to hold them. After a long exposure to the bead suspension, the amount and distribution of beads, thus, go towards an equilibrium defined by $Q, I_{x, 0}$ and the geometry of the $\mathrm{Ni}_{81} \mathrm{Fe}_{19}$ layer, as observed in Fig. 6 .

The experiments performed for varying sensor bias current $I_{x, 0}$ showed that the initial values of $-\partial V_{y, 2}^{\prime \prime} / \partial t$ were approximately independent of the value of $I_{x, 0}$, but also that the saturation level varied significantly such that more beads accumulated on the sensor for high values of $I_{x, 0}$. This shows that the electromagnetic field from the bias current only plays a small role in attracting the beads to the sensor but is important for holding beads in the capture zones near the sensor. It also shows that the efficiency and size of these capture zones increase significantly with the current. These indications are in agreement with the theoretical considerations in Section III-B2, where it was shown that the magnetic force from the bias current is localized around the sensor edges.

\section{CONCLUSION}

We have systematically investigated the capture of MyOne magnetic beads on magnetic sensors in a microfluidic channel under a fluid flow as function of the time, the bead concentration, the fluid flow rate and the sensor bias current. We have shown theoretically that the gravitational bead sedimentation flux is proportional to the bead concentration and sedimentation velocity and independent of the fluid flow profile. Experimentally, the sensor response was found to be a function of the time times the bead concentration. The initial slope of the sensor response versus time was found to be well described by a power law dependence on the fluid flow rate with a positive exponent in the investigated interval of flow rates. The measurements reveal that the gravitational sedimentation plays a major role for bringing the beads close to the sensor, whereas the magnetostatic field and the magnetic field from the sensor bias current, which are localized near the sensor edges, play an important role in stabilizing already captured magnetic beads against being torn loose by the fluid flow. This hypothesis is confirmed by experiments carried out for varying sensor bias current. The results yield an increased understanding of the bead capture mechanisms that come into play in a microfluidic channel under a fluid flow. This is important for choosing proper experimental conditions in magnetic biodetection experiments and the results also provide the experimental basis for more detailed theoretical work.

\section{REFERENCES}

[1] D. L. Graham, H. A. Ferreira, and P. P. Freitas, "Magnetoresistive-based biosensors and biochips," Trends in Biotechnol., vol. 22, pp. 455-462, 2004.

[2] M. Megens and M. Prins, "Magnetic biochips: A new option for sensitive diagnostics," J. Magn. Magn. Mater., vol. 293, pp. 702-708, 2005.

[3] G. Reiss, H. Brueckl, A. Huetten, J. Schotter, M. Brzeska, M. Panhorst, D. S. A. Becker, P. B. Kamp, A. Puehler, K. Wojczykowski, and P. Jutzi, "Magnetoresistive sensors and magnetic nanoparticles for biotechnology," J. Mater. Res., vol. 20, pp. 3294-3302, 2005.

[4] P. Freitas, R. Ferreira, S. Cardoso, and F. Cardoso, "Magnetoresistive sensors," J. Phys.: Condens. Matter, vol. 19, 2007, Article Number 165221 .

[5] S. X. Wang and G. Li, "Advances in giant magnetoresistance biosensors with magnetic nanoparticle tags: Review and outlook," IEEE Trans. Magn., vol. 44, pp. 1687-1702, 2008
[6] R. L. Edelstein, C. R. Tamanaha, P. E. Sheehan, M. M. Miller, D. R. Baselt, L. J. Whitman, and R. J. Colton, "The BARC biosensor applied to the detection of biological warfare agents," Biosens. Bioelec., vol. 14 , pp. 805-813, 2000.

[7] R. E. Thilwind, M. Megens, J. B. A. D. Van Zon, R. Coehoorn, and M W. J. Prinz, "Measurement of the concentration of magnetic nanoparticles in a fluid using a giant magnetoresistance sensor with a trench," J. Magn. Magn. Mater., vol. 320, pp. 486-489, 2008.

[8] P.-A. Besse, G. Boero, M. Demierre, V. Pott, and R. Popovic, "Detection of a single magnetic microbead using a miniaturized silicon hall sensor," Appl. Phys. Lett., vol. 80, pp. 4199-4201, 2002.

[9] L. Ejsing, M. F. Hansen, A. K. Menon, H. A. Ferreira, D. L. Graham, and P. P. Freitas, "Planar hall effect sensor for magnetic micro- and nanobead detection," Appl. Phys. Lett., vol. 84, pp. 4729-4731, 2004.

[10] C. D. Damsgaard, S. C. Freitas, P. P. Freitas, and M. F. Hansen, "Exchange-biased planar hall effect sensor optimized for biosensor applications," J. Appl. Phys., vol. 103, 2008, Article Number 07A302.

[11] B. D. Tu, T. Q. Hung, N. T. Thanh, T. M. Danh, N. H. Duc, and C. Kim, "Planar hall bead array counter microchip with NiFe/IrMn bilayers," J. Appl. Phys., vol. 104, 2008, Article Number 074701.

[12] V. Gehanno, P. P. Freitas, A. Veloso, J. Ferreira, B. Almeida, J. B Sousa, A. Kling, J. C. Soares, and M. F. Da Silva, "Ion beam deposition of Mn-Ir spin valves," IEEE Trans. Magn., vol. 35, pp. 4361-4367, 1999.

[13] G. Fonnum, C. Johansson, A. Molteberg, S. Mørup, and E. Aksnes, "Characterization of dynabeads by magnetization measurements and Mössbauer spectroscopy,” J. Magn. Magn. Mater., vol. 293, pp. 41-47, 2005.

[14] T. B. G. Hansen, C. D. Damsgaard, and M. F. Hansen, 2009, Unpublished results.

[15] A. Engel and R. Friedrichs, "On the electromagnetic force on a polarizable body," Amer. J. Phys., vol. 70, pp. 428-432, 2002.

[16] B. T. Dalslet, C. D. Damsgaard, S. C. Freitas, P. P. Freitas, and M. F. Hansen, "Bead capture and release on a magnetic sensor in a microfluidic system," Proc. IEEE Sensors 2008, pp. 242-245, 2008.

Frederik W. Østerberg received the B.S. degree from the Technical University of Denmark, Lyngby, in 2007. Currently, he is working towards the M.Sc degree at the Department of Micro and Nanotechnology, Technical University of Denmark.

Fields of interest are magnetic biosensors, and micro four-point probes used for characterization of semiconductor thin films.

Bjarke T. Dalslet received the M.S. degree from the Technical University of Denmark, Lyngby, in 2004 and the Dr. Ir. from the Universiteit Twente, Enschede, The Netherlands, in 2008.

He is currently a Postdoctoral Researcher at the Department of Micro and Nanotechnology, Technical University of Denmark. His fields of interest are production, characterization, application and design of magnetic thin-film devices and the characterization and modeling of high-temperature ceramic mixed ionic and electronic conductors.

Christian D. Damsgaard was born in Denmark in 1977. He received the M.Sc degree in engineering in 2003 and the Ph.D. degree in physics in 2006 from the Department of Physics, Technical University of Denmark, Lyngby.

In 2006, he joined Department of Micro and Nanotechnology, Technical University of Denmark, as a Postdoctoral Researcher working on developing magnetic biosensors. Since November 2008, he has been working on developing and enabling techniques for silicon-based MEMS devices.

Susana C. Freitas received the Ph.D. degree in physics from the Instituto Superior Técnico, Lisbon, Portugal, in 2002.

From 2002 to 2006, she was a Postdoctoral Fellow at INESC-MN, where she worked on magnetic thin films by ion beam deposition. Since 2003, she has been invited as an Auxiliary Professor in the Physics Department, Instituto Superior Técnico. She is presently a Senior Researcher at INESC Microsystems and Nanotechnologies. Her current research interests include thin-film deposition by ion beam, spin-dependent tunneling junctions for spin transfer devices, and magnetoresistive devices for biosensors and low magnetic field detection. 
Paulo P. Freitas received the Ph.D. degree in solid-state physics from Carnegie Mellon University, Pittsburgh, PA, in 1986, followed by a postdoctoral stay at IBM Watson Research Labs.

He is now a Full Professor at the Physics Department, Instituto Superior Tecnico, Lisbon, Portugal, and the Director of INESC Microsystems and Nanotechnologies, Lisbon. Current research topics include magnetoresistive biochips and bioelectronics, magnetoresistive sensors, MRAMS, and read heads for ultrahigh density recording.
Mikkel F. Hansen was born in 1971. He received the M.Sc. degree in physics from the University of Copenhagen, Copenhagen, Denmark, in 1995 and the Ph.D. degree in physics in 1998 from the Technical University of Denmark, Lyngby.

He then pursued post-doc positions at Uppsala University and at the Center for Magnetic Recording Research, University of California, San Diego. Since 2002, he has been an Associate Professor at the Department of Micro and Nanotechnology, Department of Micro and Nanotechnology, Technical University of Denmark. His present research interests include magnetic lab-on-a-chip systems, magnetic nanoparticles, magnetic biosensors and applications thereof. 